Type of the Paper (Case Report)

\title{
Growth Hormone (GH) Administration Increases the Metabolic Activity of the left Hippocampus in an Elder Patient with Cognitive Disorders
}

\author{
Jesús Devesa ${ }^{1 *}$, Iria Núñez ${ }^{2}$, Carlos Agra ${ }^{3}$, Alejandro Bejarano ${ }^{2}$ and Pablo Devesa ${ }^{4}$. \\ ${ }^{1}$ Scientific Direction. Medical Center Foltra. Travesía de Montouto 24. 15886-Teo. Spain; jesus.devesa@usc.es \\ ${ }^{2}$ Nuclear Medicine. Hospital HM Modelo. Virrey Osorio 30. 15011-Coruña. Spain; inunez@hmhospitales.com. \\ ${ }^{3}$ Neuropsychology. Medical Center Foltra. Travesía de Montouto 24. 15886-Teo. Spain; \\ carlosagra80@gmail.com \\ ${ }^{4}$ Research and Development. Medical Center Foltra. Travesía de Montouto 24. 15886-Teo. Spain; \\ pdevesap@gmail.com \\ * Correspondence: jesus.devesa@usc.es; Tel.: +34-981-802-928
}

\begin{abstract}
Background: We analyzed, by PET-SCAN, how growth hormone (GH) might act on the brain of a not GH-deficient elder woman who suspected that she was developing Alzheimer's disease; 2) Methods: After performing a first psychometric study (TAVEC verbal learning test), the metabolic activity of brain structures related to cognition, memory and behavior was analyzed by 18-F Fluorodeoxyglucose PET-SCAN. The patient was then treated with GH $(0.4 \mathrm{mg} / \mathrm{day})$ during three weeks and the last day under this treatment a new PET-SCAN was carried out. One month after commencing the treatment with GH a new TAVEC test was performed; 3) Results: GH administration normalized the cognitive deficits observed in the first cognitive test and significantly $(\mathrm{p}<0.025)$ increased (Voxel-Based Morphometry) the metabolic activity in the left hippocampus, left amygdala and left parahippocampus, but also in practically all brain cortical areas; 4) Conclusions: This is the first study in which the effects of GH on the brain have been visualized in images. Our data confirm the positive effects of this hormone on cognition and memories; although they do not allow us to conclude whether GH administration may be useful in the early stages of Alzheimer's disease, they seem to be promising.
\end{abstract}

Keywords: Growth Hormone, cognition, hippocampus, amygdala, parahippocampus, recent memory, PET-SCAN, Alzheimer's disease.

\section{Introduction}

Filogenetically, the limbic system is a very ancient part of the brain basically responsible for behavior, different types of memory and emotions. Structurally it is formed by different structures with diverse functions, but among those we can highlight the hippocampus, the amygdala and the parahippocampus. All of them are located bilaterally in the brain, although the main role usually corresponds to those placed in the left hemisphere. All of them receive and send a number of efferent and afferent signals to other important areas of the brain, such as the temporal and prefrontal lobes.

In the case of the hippocampus, it has been demonstrated that it is an important neurogenic niche, where adult neurogenesis takes place in humans [1]. Functionally, the hippocampus is responsible for the acquisition of recent memory, learning, and spatial orientation and navigation (in neurons known as place cells). These functions are affected in personality disorders, perhaps because of long-term glucorticoid excess [2], which negatively regulates the formation of new neurons in the subgranular zone (SGZ) of the gyrus dentatus of the hippocampus [3].

The amygdala is involved in emotional responses (pleasure, fear, anger, anxiety), and also determines how emotions are attached to memories, mainly forming new memories related to fear, although a recent paper describes that fear is the result of a very complex fear memory network [4].

Regarding the parahippocampus, a recent study describes that, in humans, its posterior section is involved in the visuospatial perception, while the anterior section is related to mnemonic processes, 
therefore suggesting that this structure acts as a functional interface between perception and memories [5].

25 years ago it was discovered that growth hormone $(\mathrm{GH})$ receptor gene was expressed in many different areas of the CNS of rats and rabbits [6]. This finding led to the assumption that GH should perform important functions at the central level, as indicated by the psychological improvements observed in adult patients treated with the hormone [7], particularly improving memory and cognition [8]. On the contrary, untreated GH-deficient (GHD) adults usually show significant psychological affectations related to lack of energy, memory and cognitive alterations [9].

GH plays a key role during the development of the CNS [10], and the hormone has been found to be produced within the postnatal hippocampus in rats [11-12], where GH administration increases the proliferation of stem cells in normal adult rats [13]; this also occurs when the hormone is given after a brain injury produced by kainic acid administration, perhaps by cooperating with the GH endogenously expressed, as its receptor, in hippocampal progenitor cells [14]. Moreover, memory tasks induce synthesis of GH in mice hippocampus leading to the appearance of newly formed neurons [8].

The effects of GH on cognitive functions in humans have been widely reviewed recently [15-16].

For all this, in this study, we tried to visualize how GH treatment might act on brain areas involved in cognition in an elder woman who began to show cognitive deficits. Our results clearly indicate that short-term GH treatment produced a clear increase in the metabolic activity of the left hippocampus and amygdala, and also in the left parahippocampus, structures that previously showed a hypometabolism, as revealed by a PET-SCAN. The improvements observed after GH treatment were also confirmed by the TAVEC verbal learning test.

\section{Results}

\subsection{Cognitive test: TAVEC test}

Most of the scores registered as deficients in the first test changed to the mean for anormal population during the second test performed. This indicates a positive learning curve as well as an increased attention. Moreover, the index of discriminability indicates learning by the patient, because in the second test she was already capable of storing the information discriminately. In addition, during the second test carried out there was not loss of information (fading) over time.

These data indicate the treatment with GH induced a clear improvement in learning, attention and memory.

The main scores of this test are shown in Table 1.

\begin{tabular}{|c|c|c|c|}
\hline Assay & Maximal score & First test & Second Test \\
\hline Immediate free memory & 80 & 36 & 49 \\
\hline Short-term free memory & 16 & 9 & 12 \\
\hline Long-term free memory & 16 & 8 & 12 \\
\hline Long-term recognition & 16 & $10\left({ }^{*} 4\right)$ & $16\left({ }^{*} 2\right)$ \\
\hline
\end{tabular}

Table 1.- Main items in the TAVEC test. Values in the first and second test correspond to the number of correct answers. While scores in the first test ranged below the mean of the normal subjects, the second test indicated that the results obtained were now between the range of normal subjects. Indicates the number of false positives in this assay.

\subsection{PET-SCAN studies}

The first PET-SCAN indicated significant tracer uptake deficits in the left hippocampus, left amygdala, left parahippocampus and the subgenual area of the anterior cingulate of the right hemisphere; in each case, clearly asymmetric with respect to the metabolic activity in the other hemisphere. These deficits were 1.5 standard deviations (SD) lower than the mean value recorded in the database of the normal population (Figure 1). 

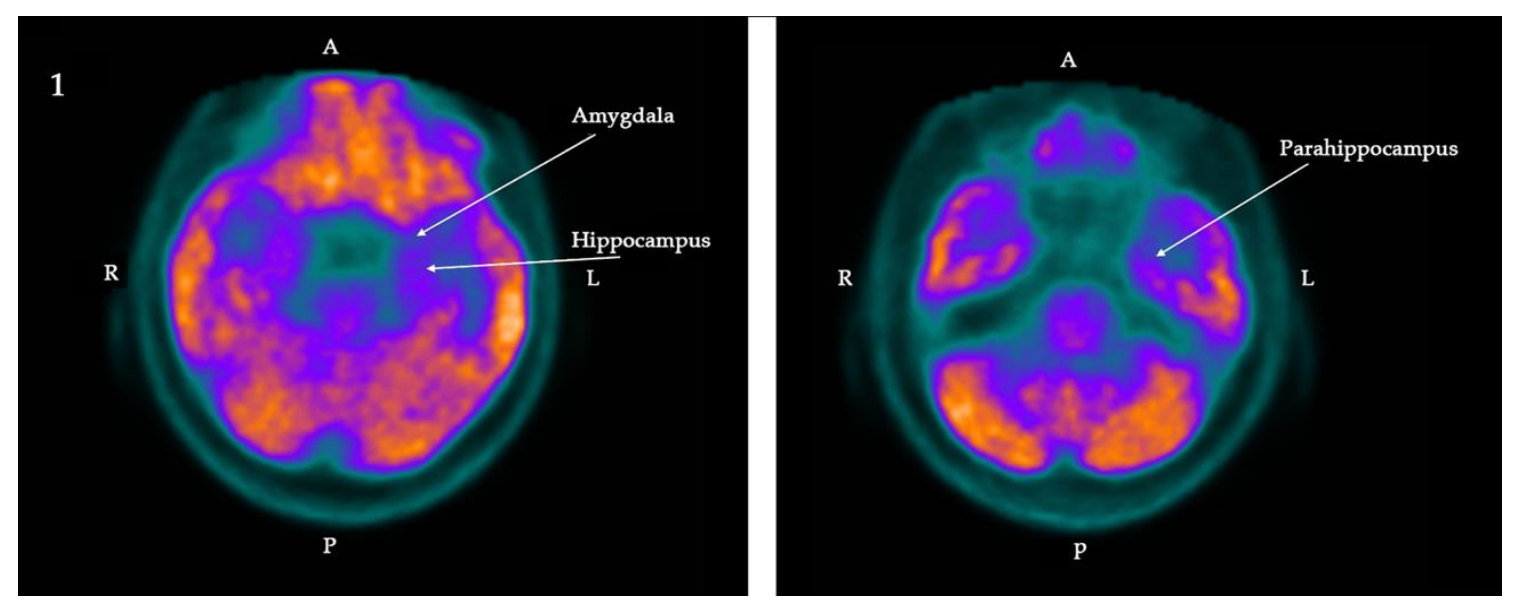

Figure 1.- Transversal section of the brain showing the metabolic activity in left amygdala, left hippocampus and left parahippocampus in the first (1) PET-SCAN study (basal conditions). Note the low metabolic activity in these structures. A: Anterior. R: Right. L: Left. P: Posterior.

These hypometabolisms were normalized after the short-term treatment with $\mathrm{GH}$, as the second PET-SCAN indicates (Figure 2), not only in the areas specifically analyzed but also in most of the cortical brain structures in both hemispheres.
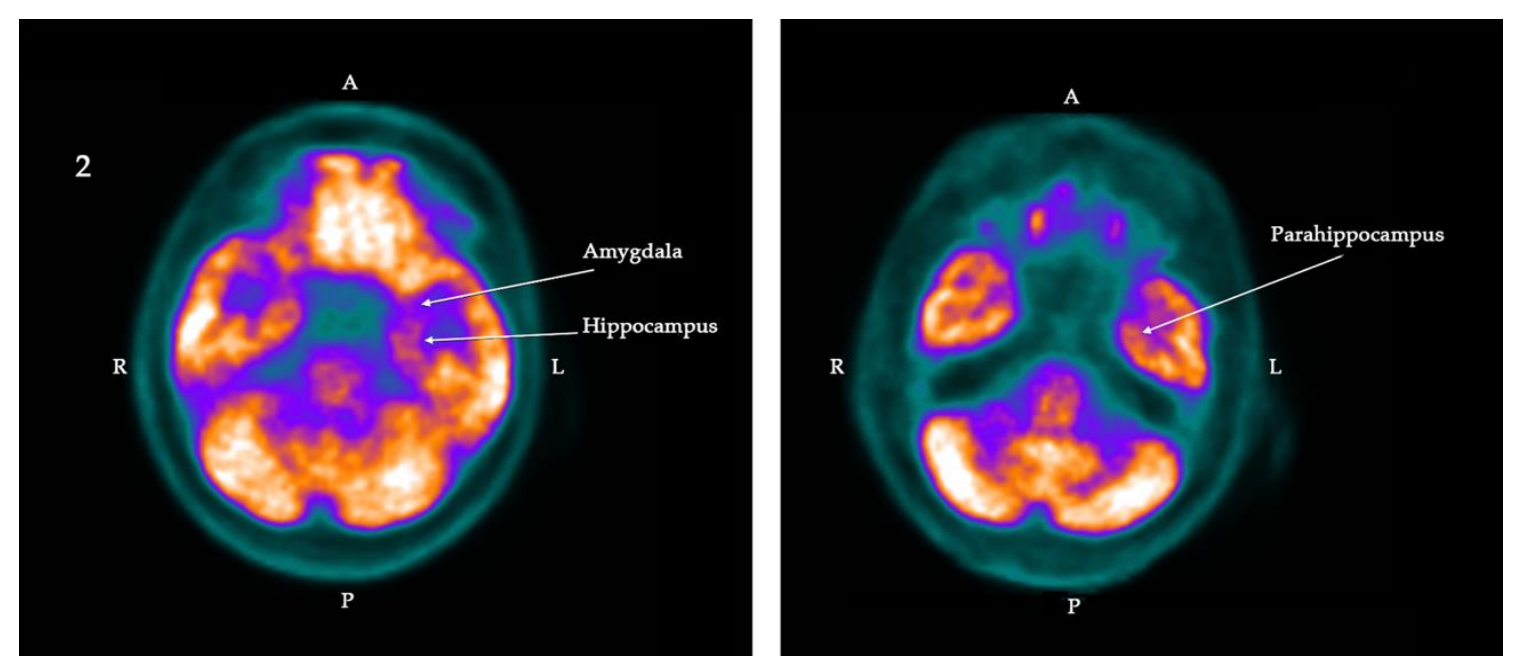

Figure 2.- Transversal section of the brain showing the metabolic activity in left amygdala, left hippocampus and left parahippocampus in the second (2) PET-SCAN study (after 3-weeks receiving GH treatment). The low metabolic activity previously observed in these structures significantly increased $(p<0.025)$ after GH treatment. Also note the higher metabolic activity in practically all cortical areas. A: Anterior. R: Right. L: Left. P: Posterior.

The metabolic activity of the subgenual area of the anterior cingulate of the right hemisphere also increased significantly $(\mathrm{P}<0.025)$ after $\mathrm{GH}$ treatment, being then abssolutely normal (data not shown).

\subsection{Blood analysis.}

The pre-treatment blood analysis showed that the patient had a dyslipidemia (plasma total cholesterol values: $275 \mathrm{mg} / \mathrm{dl}$; plasma triglycerides: $195 \mathrm{mg} / \mathrm{dl}$. Normal values: 110-200 and 50-150, respectively). Erythrocytes and $\mathrm{Hb}$ were in normal values, as they were plasma glucose, proteins, liver transaminases and tumoral markers (CA-125, CA15-3, CA19-9, Alpha-fetoprotein and CEA). 
Plasma thyroid-stimulating hormone (TSH) was normal $(2.18 \mu \mathrm{UI} / \mathrm{ml})$, as was free thyroxine (fT4, 1.1 $\mathrm{ng} / \mathrm{dl})$; plasma cortisol at 8 a.m. was also normal ( $24 \mu \mathrm{g} / \mathrm{dl})$. Plasma IGF-1 value and plasma insulin like growth factor binding protein 3 (IGFBP3) were also in normal values for the sex and age of the patient $(125 \mathrm{ng} / \mathrm{ml}$ and $2.8 \mu \mathrm{g} / \mathrm{ml}$, respectively). An intravenous arginine hydroclhoride test demonstrated that the patient was not GHD (GH peak value: $4.6 \mathrm{ng} / \mathrm{ml})$.

The blood analysis carried out after 1-month of GH treatment indicated that not significant changes existed, excepting that plasma IGF-1 and plasma IGFBP3 values increased to $185 \mathrm{ng} / \mathrm{ml}$ and $3.2 \mu \mathrm{g} / \mathrm{ml}$, respectively (both in normal values). Glycemia did not change because of short-term GH treatment, remaining in normal values.

Treatment with GH did not produce any adverse side effects.

\section{Discussion}

In this study we demonstrate for the first time, with PET-SCAN brain images, the positive effect that GH exerts on the human brain in a not GHD patient, particularly in areas related with cognition, recent memory, behavior and visuospatial perception, although, as stated above, the effects of the hormone took place in practically all cortical areas (see Figures 1 and 2).

Most likely because of health laws prohibiting the use off-label of GH in most first world countries, there are few studies showing that GH aministration increases cognition in not GHD human patients with cognitive deficits occurring as a consequence of different pathologies [17-21].

Of high interest here is the fact that the low metabolic activity observed in the left hippocampus, left amygdala and left parahippocampus, was significantly increased after the short-term treatment with GH. This increased metabolic activity cannot be attributed to a higher availability of glucose, the main nutrient for neurons, produced by the previous treatment with GH. Although this hormone slightly increases glycemia, and this is the reason by which GH is one of the four counterregulatory hormones opposed to insulin effects, the acute increase in glycemia after GH administration is small in magnitude and plasma glucose is rapidly taken up by muscles. Moreover, if glucose was the responsible factor of the increased metabolic activity observed in those brain structures, they would not have captured FDG, and no changes could have been seen in the second PET-SCAN performed.

Two main possible explanations can justify our data: 1) GH induced adult neurogenesis; 2) GH increased the number and/or lenght of connections of pre-existing neurons.

In humans, adult neurogenesis takes place in some known neurogenic niches (among them the gyrus dentatus of the hippocampus) [1], although very recently it has been reported that neurogenic precursors cells also exist in the basolateral amygdala of adult mice giving origin to newly formed interneurons [22]. However adult neurogenesis in the human amygdala remains to be proved.

Recently, it was published that hippocampal neurogenesis begins to decrease abruptly from adolescence to undetectable in adults [23]. This led to a high controversy and very recent publications question or contradict these postmortem findings [24-26], although the possibility exists that adult hippocampal neurogenesis might be dysregulated by neurological diseases, such as epilepsy, or behavior disorders [27], therefore explaining the controversy currently existing about the persistence of human adult neurogenesis throughout life. Data from this study may contribute to clarify these controverses. If we could have used 3'-deoxy-3'-[18F]fluoro-L-thymidine instead of FDG we could have detected if the changes observed in the PET-SCAN were or were not due to the neurogenesis of the adult, as a study analyzed in adult rats [28].

The second possibility is that GH induced sprout of dendritic spines and changes in the lenght and density of pre-existing dendrites in the hippocampus, as it has been demonstrated after intracerebroventricular administration of the hormone in adult rats [29]. However, this possibility does not exclude the one indicated above.

Another point of interest in our study is that hippocampal atrophy (as measured by MRI) is an early marker of Alzheimer's disease that correlates with affected memory functions [30], and glucose hypometabolism has been found to be significantly reduced in early-stages of Alzheimer's disease [31]. This correlates with the reduced metabolic activity that we found in this structure in our patient 
before being treated with GH. However, if this means that this patient is developing an Alzheimer's disease, the fact that GH treatment fully corrected the abnormalities initially observed, and her quality of life improved, seems to indicate that treatments with this hormone in early stages of this disease may be of great utility, at least during some time for decreasing the progression of the disease or stopping it. Further studies are needed in a larger number of patients to check this assumption, also postulated in a previous review [32].

The results obtained in the TAVEC test corroborate the positive effects of GH observed in the brain imaging studies, although a limitation of this verbal learning test is, in this case, that only one month elapsed between the first and second tests, when it is usual to carry it out with a one-year interval for avoiding the possibility of learning the answers after the first test.

The possibility exists that GH induced the local expression of IGF-1, as it has been demonstrated in the human fetal cortex [33], but not in human adult brain. In fact, an important role for IGF-1 in neurodegenerative diseases has been postulated long time ago [34], and recently it has been published that IGF-1 increases hippocampal neurogenesis and improves memory in old patients [35]. Even more, it has been postulated that treatment with this peptide might be useful in neurodegenerative diseases, including Alzheimer's disease [36]. However, differently to that occurring with GH, plasma IGF-1 hardly crosses the blood-brain barrier. Therefore, if the effects we observed in this study were produced by IGF-1, this must have occurred by the induction exerted by $\mathrm{GH}$ of the synthesis of that peptide in the brain.

Another possibility for explaining the findings we observed is that GH has produced an increase in brain flow in the patient, allowing greater uptake of FDG. Recently, we described that GH induces an important reparative effect on the endothelial dysfunction that appears after atherogenic stimuli, such as hypercholesterolemia [37]; moreover, the hormone is a mitochondrial protector [38-39], and atherogenesis is related to oxidative stress. Therefore, since the patient we treated had high levels of plasma cholesterol and triglycerides it is also possible that, despite the short time of treatment, GH may have contributed to improve the blood supply to the brain, facilitating the appearance of the changes here described (both in terms of PET-SCAN images and cognitive tests).

In summary, for the first time we proved with brain images that GH exerts positive effects on the brain. Moreover, according to our unpublished data in a high number of patients with acquired neurological injuries, GH treatment is safe if given at low doses and during short periods of time, although the use of GH "off-label" does not allow us to publish more than case by case at any time.

\section{Materials and Methods}

The patient was a 61 year old woman, caucasic, whose father had died of a very aggressive Alzheimer's disease at 64 years of age. The patient has 4 sons, she works as director of a company. Among her clinical antecedents stand out hysterectomy because of a fibromyoma and familial dyslipidemia. She was no taking other medication than melatonin at night.

At admission in our Medical Center, the patient referred that in the last two years she was suffering from an important stress, due to labor problems, a decrease in recent memory, sporadic episodes of temporo-spatial disorientation and occasions of disruptive behavior. Due to the disease suffered by her father, she was afraid that an Alzheimer's disease was beginning to be developed also in her.

Clinical examinations were normal. Blood pressure was 135/70 $\mathrm{mm} \mathrm{Hg}$. The body mass index (BMI) was normal: $23 \mathrm{~kg} / \mathrm{m}^{2}$. Blood analysis (hematimetry, biochemistry, thyroid hormones, cortisol, IGF-1, IGFPB3 and tumoral markers) were carried out before and one month after starting with GH treatment. To assess the possibility of an existing adult GHD, during the first blood analysis a typical arginine test (30 g arginine hydrochloride was given as an intravenous infusion from 0-30 min, and samples for analyzing plasma GH levels were withdrawn at 0, 30, 60, 90 and $120 \mathrm{~min}$ ).

\subsection{Cognitive tests}

Initially a TAVEC verbal learning test was performed. This test is based on other similar psychometric tests, such as the Test of 15 words of Rey or the most recent CVLT-California Verbal 
Learning Test. In all of them, verbal element learning test are used. In the TAVEC test specifically 3 lists are used for Learning, Interference and Recognition. The test allows to establish the normality of the patient (as compared with a sample similar in age, sex, and educative level), and to describe the functioning of the patient's memory and to determine the form and reasons for its deviation (if any exists).

One month after the first test, a new TAVEC test was performed.

\subsection{PET-SCANS}

The next day, after the first TAVEC test, the metabolic activity of the brain of the patient was analyzed by PET-SCAN imaging in coronal, sagittal and transversal sections obtained 30 minutes after administering $4.2 \mathrm{mCi}$ 18-F Fluorodeoxyglucose (FDG), correcting attenuation with CT-SCAN images. One day later, and once obtained signed informed consent, the patient began a treatment with GH ( $0.4 \mathrm{mg} /$ day, s.c., at 10.00 am; Nutropín, Ipsen, Spain).

21 days later a new PET-SCAN was carried out in the same conditions, excepting that the dose of FDG administered was now slightly lower (3.6 mCi).

Last GH administration took place 1 hour before the second PET-SCAN was performed. Both PET-SCAN were performed at $11.00 \mathrm{am}$.

In both cases the patient was fasting for 14 hours before the exams. One month after the first test a new TAVEC verbal learning test was carried out.

In both PET-SCAN studies a quantitative analysis between the metabolism in brain areas located in both hemispheres was carried out by Voxel-Based Morphometry by using the software Neurocloud PET. The same technique allowed to compare the metabolic differences in the same brain areas between the first and second PET-SCAN.

Studies and treatment were conducted according to the protocols of the Medical Center Foltra in compliance with with national legislation for the use of GH off-label and the Code of Ethics of the World Medical Association (Declaration of Helsinki).

Signed informed consent was obtained from the patient for publishing the results obtained.

\subsection{Statistical analysis}

The statistical significance of the quantitative analysis (voxel-to-voxel) between the first and the second PET-SCAN studies was established by two-sample- $t$-test. This test was also used for carrying out a parametric analysis between data obtained in the patient and the database of normal subjects. A $P$ value $<0.05$ was considered to be significant. Statistical analysis was blind and carried out by an external company specialized in Voxel-Based Morphometry techniques.

\section{Conclusions}

Results from this study clearly show, for the first time in human patients, that GH exerts a strong and positive effects on cortical brain structures, being of special interest the fact that the hormone is able to increase the metabolic activity in structures related to cognition and memories. The possibility exists that GH treatment in the early-stages of Alzheimer's disease can be useful to slow down the progression of this disease and/or other neurodegenerative disorders that lead to cognitive impairments.

Author Contributions: "Conceptualization, J.D. and P.D.; Methodology, J.D., I.N., C.A., A.B. and P.D.; Software, I.N. and A.B.; Validation, J.D., I.N., C.A.; Formal Analysis, J.D., I.N., C.A.; Investigation, J.D. and P.D.; WritingOriginal Draft Preparation, J.D.; Writing-Review \& Editing, J.D. and I.N.; Supervision, J.D.; Project Administration, J.D.; Funding Acquisition, J.D.".

Funding: "This research was funded by Foundation Foltra grant number [2018-02]."

Acknowledgments: Voxel-Based Morphometry was performed by Qubiotech Health Intelligence S.L. (Coruña. Spain). 
Conflicts of Interest: "The authors declare no conflict of interest." "The founding sponsors had no role in the design of the study; in the collection, analyses, or interpretation of data; in the writing of the manuscript, and in the decision to publish the results".

\section{References}

1. Eriksson, P.S.; Perfilieva, E.; Björk-Eriksson, T.; Alborn, A.M.; Nordborg, C.; Peterson, D.A., et al. Neurogenesis in the adult human hippocampus. Nat Med 1998; 4:1313-7.

2. Driessen, M.; Herrmann, J.; Stahl, K.; Zwaan, M.; Meier, S.; Hill, A., et al. Magnetic resonance imaging volumes of the hippocampus and the amygdala in women with borderline personality disorder and early traumatization. Arch Gen Psychiatry 2000; 57: 1115-22.

3. Gould, E.; McEwen, B.S.; Tanapat, P.; Galea, L.A.; Fuchs, E. Neurogenesis in the dentate gyrus of the adult tree shrew is regulated by psychosocial stress and NMDA receptor activation. J Neurosci 1997; 17:2492-8.

4. Vetere, G.; Kenney, J.W.; Tran, L.M.; Xia, F.; Steadman, P.E.; Parkinson, J., et al. Chemogenetic Interrogation of a Brain-wide Fear Memory Network in Mice. Neuron 2017; 94:363-74.

5. Baumann, O.; Mattingley, J.B. Functional Organization of the Parahippocampal Cortex: Dissociable Roles for Context Representations and the Perception of Visual Scenes. J Neurosci 2016; 36:2536-42.

6. Lobie, P.E.; García-Aragón, J.; Lincoln, D.T.; Barnard, R.; Wilcox, J.N.; Waters, M.J. Localization and ontogeny of growth hormone receptor gene expression in the central nervous system. Brain Res Dev Brain Res 1993; 74:225-33.

7. Nyberg, F.; Burman, P. Growth hormone and its receptors in the central nervous system--location and functional significance. Horm Res 1996; 45:18-22.

8. Waters, M.J.; Blackmore, D.G. Growth hormone (GH), brain development and neural stem cells. Pediatr Endocrinol Rev 2011; 9:549-53.

9. Jørgensen, J.O.; Vahl, N.; Hansen, T.B.; Thuesen, L.; Hagen, C.; Christiansen, J.S. Growth hormone versus placebo treatment for one year in growth hormone deficient adults: increase in exercise capacity and normalization of body composition. Clin Endocrinol (Oxf) 1996; 45:681-8.

10. Lobie, P.E.; Zhu, T.; Graichen, R.; Goh, E.L. Growth hormone, insulin-like growth factor I and the CNS: localization, function and mechanism of action. Growth Horm IGF Res 2000;10 Suppl B: S51-6.

11. Donahue, C.P.; Jensen, R.V.; Ochiishi, T.; Eisenstein, I.; Zhao, M.; Shors, T., et al. Transcriptional profiling reveals regulated genes in the hippocampus during memory formation. Hippocampus 2002; 12:621-33.

12. Donahue, C.P.; Kosik, K.S.; Shors, T.J. Growth hormone is produced within the hippocampus where it responds to age, sex, and stress. Proc Natl Acad Sci U S A 2006; 103:6031-6.

13. David Aberg, N.; Lind, J.; Isgaard, J.; Georg, K.H. Peripheral growth hormone induces cell proliferation in the intact adult rat brain. Growth Horm IGF Res 2010; 20:264-9.

14. Devesa, P.; Reimunde, P.; Gallego, R.; Devesa, J.; Arce, V. Growth hormone (GH) treatment may cooperate with locally-produced GH in increasing the proliferative response of hippocampal progenitors to kainateinduced injury. Brain Inj 2011; 25:503-10.

15. Nyberg, F.; Hallberg, M. Growth hormone and cognitive function. Nat Rev Endocrinol 2013; 9:357-65.

16. Devesa, J.; Almengló, C.; Devesa, P. Multiple Effects of Growth Hormone in the Body: Is it Really the Hormone for Growth? Clin Med Insights Endocrinol Diabetes 2016; 9:47-71.

17. Song, J.; Park, K.; Lee, H.; Kim, M. The effect of recombinant human growth hormone therapy in patients with completed stroke: a pilot trial. Ann Rehabil Med 2012; 36:447-57.

18. Devesa, J.; Reimunde, P.; Devesa, P.; Barberá, M.; Arce, V. Growth hormone (GH) and brain trauma. Horm Behav 2013; 63:331-44.

19. Devesa, J.; Díaz-Getino, G.; Rey, P.; García-Cancela, J.; Loures, I.; Nogueiras, S., et al. Brain Recovery after a Plane Crash: Treatment with Growth Hormone (GH) and Neurorehabilitation: A Case Report. Int J Mol Sci 2015; 16:30470-82.

20. Devesa, J.; Lema, H.; Zas, E.; Munín, B.; Taboada, P.; Devesa, P. Learning and Memory Recoveries in a Young Girl Treated with Growth Hormone and Neurorehabilitation. J Clin Med 2016; 5: pii:E14.

21. Quintana, A.; Agra, C.; Outeiral, L.; Devesa, A.; Llorente, D.; Devesa, J. Cognitive Evolution of a Patient Who Suffered a Subarachnoid Haemorrhage Eight Years Ago, after Being Treated with Growth Hormone, Melatonin and Neurorehabilitation. Reports 2018; 1, 2. 
22. Jhaveri, D.J.; Tedoldi, A.; Hunt, S.; Sullivan, R.; Watts, N.R.; Power, J.M., et al. Evidence for newly generated interneurons in the basolateral amygdala of adult mice. Mol Psychiatry 2018; 23:521-32.

23. Sorrells, S.F.; Paredes, M.F.; Cebrian-Silla, A.; Sandoval, K.; Qi, D.; Kelley, K.W., et al. Human hippocampal neurogenesis drops sharply in children to undetectable levels in adults. Nature 2018; 555:377-81.

24. Lee, H.; Thuret, S. Adult Hippocampal Neurogenesis: Controversy and Evidence. Trends Mol Med 2018; pii: S1471-4914(18)30079-0.

25. Kempermann, G.; Gage, F.H.; Aigner, L.; Song, H.; Curtis, M.A.; Thuret, S., et al. Human Adult Neurogenesis: Evidence and Remaining Questions. Cell Stem Cell 2018; pii: S1934-5909(18)30166-8.

26. Boldrini, M.; Fulmore, C.A.; Tartt, A.N.; Simeon, L.R.; Pavlova, I.; Poposka, V. et al. Human Hippocampal Neurogenesis Persists throughout Aging. Cell Stem Cell 2018; 22:589-99.

27. Toda, T.; Parylak, S.L.; Linker, S.B.; Gage, F.H. The role of adult hippocampal neurogenesis in brain health and disease. Mol Psychiatry 2018; [Epub ahead of print].

28. Tamura, Y.; Kataoka, Y. PET imaging of neurogenic activity in the adult brain: Toward in vivo imaging of human neurogenesis. Neurogenesis (Austin) 2017; 4: e1281861.

29. Olivares-Hernández, J.D.; García-García, F.; Camacho-Abrego, I.; Flores, G.; Juárez-Aguilar, E. Intracerebroventricular administration of growth hormone induces morphological changes in pyramidal neurons of the hippocampus and prefrontal cortex in adult rats. Synapse 2018; [Epub ahead of print].

30. Petersen, R.C.; Jack, C.R. Jr.; Xu, Y.C.; Waring, S.C.; O’Brien, P.C.; Smith, G.E., et al. Memory and MRIbased hippocampal volumes in aging and AD. Neurology 2000; 54:581-7.

31. Choi, E.J.; Son, Y.D.; Noh, Y.; Lee, H.; Kim, Y.B.; Park, K.H. Glucose Hypometabolism in Hippocampal Subdivisions in Alzheimer's Disease: A Pilot Study Using High-Resolution ${ }^{18}$ F-FDG PET and 7.0-T MRI. J Clin Neurol 2018; 14:158-64.

32. Bianchi, V.E.; Locatelli, V.; Rizzi, L. Neurotrophic and Neuroregenerative Effects of GH/IGF1. Int J Mol Sci 2017; 18. pii: E2441.

33. Pathipati, P.; Gorba, T.; Scheepens, A.; Goffin, V.; Sun, Y.; Fraser, M. Growth hormone and prolactin regulate human neural stem cell regenerative activity. Neuroscience 2011, 190:409-27.

34. Dore, S.; Kar, S.; Quirion, R. Rediscovering an old friend, igf-i: Potential use in the treatment of neurodegenerative diseases. Trends Neurosci 1997; 20: 326-331.

35. Morel, G.R.; Leon, M.L.; Uriarte, M.; Reggiani, P.C.; Goya, R.G. Therapeutic potential of IGF-1 on hippocampal neurogenesis and function during aging. Neurogenesis (Austin) 2017, 4, e1259709.

36. Feldman, E.L.; Sullivan, K.A.; Kim, B.; Russell, J.W. Insulin-like growth factors regulate neuronal differentiation and survival. Neurobiol Dis 1997, 4: 201-14.

37. Caicedo, D.; Díaz, O.; Devesa, P.; Devesa, J. Growth Hormone (GH) and Cardiovascular System. Int J Mol Sci 2018; 19. pii: E290.

38. Keane, J.; Tajouri, L.; Gray, B. The effect of growth hormone administration on the regulation of mitochondrial apoptosis in-vivo. Int J Mol Sci 2015; 16:12753-72.

39. Nylander, E.; Grönbladh, A.; Zelleroth, S.; Diwakarla, S.; Nyberg, F.; Hallberg, M. Growth hormone is protective against acute methadone-induced toxicity by modulating the NMDA receptor complex. Neuroscience 2016; 339:538-47. 